\title{
PROSPECTIVE RANDOMIZED CONTROL TRIAL OF ULTRASOUND GUIDED INTERNAL JUGULAR VENOUS CANNULATION: THE SHORT VERSUS THE LONG AXIS APPROACH.
}

1. MBBS, FCPS

Associate professor Anaesthesia AIMC, Jinnah Hospital, Lahore.

2. MBBS, FCPS

Senior registrar Anaesthesia

Burn Unit Jinnah Hospital, Lahore.

3. MBBS, FCPS

Assistant professor Anaesthesia

AIMC, Jinnah Hospital, Lahore.

4. MBBS, FCPS

Senior registrar Anaesthesia

AIMC, Jinnah Hospital, Lahore.

5. MBBS, FCPS

Senior registrar, Anaesthesia Kaul Associates; Lahore.

6. MBBS, FCPS

Professor Anaesthesia

AIMC, Jinnah Hospital, Lahore.

Correspondence Address:

Dr. Liaqat Ali

Associate professor Anaesthesia

AIMC, Jinnah Hospital, Lahore.

aliliaqatdoc@gmail.com

Article received on:

30/08/2019

Accepted for publication:

15/01/2020

\author{
Liaqat Ali ${ }^{1}$, Shaheer Nayyar ${ }^{2}$, Rizwan Ahmad Khan ${ }^{3}$, Hajra Shuja ${ }^{4}$, Irfan Liaqat Ali ${ }^{5}$, \\ Muhammad Ashraf $\mathrm{Zia}^{6}$
}

ABSTRACT... Objectives: To compare the first pass success rate between long and short axis approaches in ultrasound guided IJV cannulation. Study Design: Prospective randomized controlled study. Setting: Department of Anesthesia and Intensive Care, Jinnah Hospital, Lahore. Period: Between $3^{\text {rd }}$ Mar, 2016 to $2^{\text {nd }}$ Sep, 2016. Material \& Method: One hundred and ten patients admitted in the ICU were included in this study. Internal jugular vein was visualized ultrasonographically. In group A patients, long-axis view was used, while in group B patients, short-axis view was used to pass the needle. Under aseptic measures, US probe was placed on the neck and IJV viewed with either approach. During needle advancement if the needle penetrates the vein in the first attempt and blood flush-back is viewed, it was labeled as $1^{\text {st }}$ pass success. This first pass success rate was noted. Results: One hundred and ten patients (55 in each group) were included in the study. The mean age of the patients was found to be $33.89 \pm$ 13.35 years in group $A$ and $35.90 \pm 14.10$ years in group $B$. The primary outcome of the study was to compare $1^{\text {st }}$ pass success rate in both groups. The first pass success rate was $74.5 \%$ in group B (short-axis) and 61.8\% in group A (long-axis); independent sample test showed nonsignificant $(P=0.219)$. There is no difference in the frequency of $1^{\text {st }}$ pass success rate in short axis approach and long axis approach for Ultrasound-guided IJV cannulation. Conclusion: Ultrasound-guided internal jugular venous cannulation with both approaches i.e. short-axis view and long-axis view have no significant difference in terms of first pass and therefore are equally successful.

Key words: Central venous line; long-axis; short-axis, in-plane, out-of-plane, Ultrasonography.

Article Citation: Ali L, Nayyar S, Khan RA, Shuja H, Ali IL, Zia MA. Prospective randomized control trial of ultrasound guided internal jugular venous cannulation: The short versus the long axis approach. Professional Med J 2020; 27(2):407412. DOI: 10.29309/TPMJ/2020.27.2.4450

\section{INTRODUCTION}

Internal Jugular Vein (IJV) cannulation is a commonly performed procedure in hemodynamically unstable patients particularly those admitted in intensive care units (ICU). The main purpose of IJV cannulation is hemodynamic monitoring, long term administration of fluids, antibiotics, nutrition and hemodialysis. Over the last decade use of IJV cannulation has increased worldwide $^{1}$ due to lesser complications than subclavian vein cannulation.

US-guided cannulation of IJV has gained tremendous success because of it being associated with increased success rate and lower rate of complications than the landmark technique. US can facilitate direct visualization of
IJV, its dimensions, orientation and surrounding structures making it a safe procedure. US-guided cannulation can significantly reduce the risk of complications such as pneumothorax, arterial puncture, hematoma, hemodynamic instability, local site infection, pain, vasovagal reflexes etc. The National Institute for Clinical Excellence (NICE) published guidelines supporting the routine use of ultrasound guidance for internal jugular vein cannulation and also by recommendations of the American Society of Echocardiography and the society of Cardiovascular Anesthesiologists. ${ }^{2}$

There are two approaches to ultrasonography (USG) for insertion of IJV cannula, the shortaxis (SAX) and long-axis (LAX) transducer approaches. Various studies demonstrated 
that SAX view is potentially superior due to its significantly higher success rate in terms of first pass. ${ }^{3}$ However another study demonstrated that LAX approach for IJV cannulation was more efficient than SAX, with LAX view associated with decrease in number of redirections at IJV and SC sites. $^{4}$

\section{METHODOLOGY}

In this randomized controlled trial carried out at department of Anesthesia and Intensive Care, Jinnah Hospital, Lahore during the period 03-032016 to 02-09-2016, 110 patients were enrolled via non probability purposive sampling. Approval from institutional Ethics Committee was obtained for the study.

Patients aged 18 - 60 years admitted in ICU with the prescription to get IJV catheter were included. Exclusion criteria were patients having platelet count $<100,000 / \mathrm{mm}^{3}$, patients with deranged coagulation profile (PT $>6 \mathrm{sec}$ than control and APTT 1.3 times), patients with inflammation or infection (pus) over cannula insertion site on examination, and evidence of jugular vein thrombosis (on USG).

All the study related information were collected on a pre-designed proforma and included the demographic details. By lottery technique patients were divided into two groups. Ultrasound-guided IJV view in fifty five patients in group A was done by long-axis approach and fifty five patients by shortaxis approach.

All the patients in both groups were in supine position with neck tilted towards the opposite side. Standard monitoring i.e. ECG (rate and rhythm), NIBP, $\mathrm{SpO}_{2}$ were in place. Under aseptic measures, US probe (linear $12 \mathrm{~Hz}$; Mindray Z6 Color doppler) was placed on the neck and IJV cannulation was obtained by either of the two approaches. IJV was viewed with either approach and needle will be advanced. If the needle penetrates the vein in the first attempt and blood flush-back is viewed, it was labeled as $1^{\text {st }}$ pass success. Central lines were placed by senior anesthetist having experience of at least one year in USG techniques. If needle didn't pass first time, then needle was withdrawn and passed again and it was noted. After passing the needle into the IJV, guide wire and then central venous catheter was passed into the vein and secured.

All the collected data was entered into SPSS version 21.0 and analyzed. Mean and standard deviation was calculated for age, height, weight and BMI of the patients. Qualitative variables such as gender and first-pass success rate were presented as frequency and percentages. First-pass success rate was considered in both groups using chi-square test. Effect modifiers were stratified and independent sample t-test was used for quantitative variables and chi square for qualitative variables and post stratification $p$ value $\leq 0.05$ was taken as significant.

\section{RESULTS}

A total of 110 patients ( $n=55$ in each group) were included in the study. The mean age of the patients was $33.89 \pm 13.35$ years in group $A$ and $35.90 \pm 14.10$ years in group B. Patients were further categorized according to age groups into 2 groups. The distribution of patients according to age is summarized in Table-l.

\begin{tabular}{|l|c|c|c|c|}
\hline \multirow{2}{*}{ Gender } & \multicolumn{2}{|c|}{$\begin{array}{c}\text { Group-A } \\
(\mathbf{n = 5 5 )}\end{array}$} & \multicolumn{2}{c|}{$\begin{array}{c}\text { Group-B } \\
(\mathbf{n = 5 5 )}\end{array}$} \\
\cline { 2 - 5 } & $\mathbf{n}$ & $\%$ & $\mathbf{n}$ & $\%$ \\
\hline 18-40 Years & 40 & $72.7 \%$ & 38 & $69 \%$ \\
\hline 41-60 Years & 15 & $27.2 \%$ & 17 & $31 \%$ \\
\hline Total & 55 & 100 & 55 & 100 \\
\hline Mean \pm SD & $33.89 \pm 13.35$ years & $35.90 \pm 14.10$ years \\
\hline
\end{tabular}

Gender distribution of the patients showed that in group $A$, most of patients were female while in group $B$, most of them were male as summarized in Table-II. It is given in Table-III along with height and weight of patients in both groups.

The primary outcome of the study was to compare $1^{\text {st }}$ pass success rate in both groups. It was found that $1^{\text {st }}$ pass success rate was $74.5 \%$ in group B (short-axis) and $61.8 \%$ in group A (longaxis) and P-value was found to be significant ( $p$ $<0.05$ ) (Table-IV). Hence there was no significant 
difference between the two approaches for $1^{\text {st }}$ pass success rate. Also $1^{\text {st }}$ pass success rate was stratified according to age, gender and BMI.

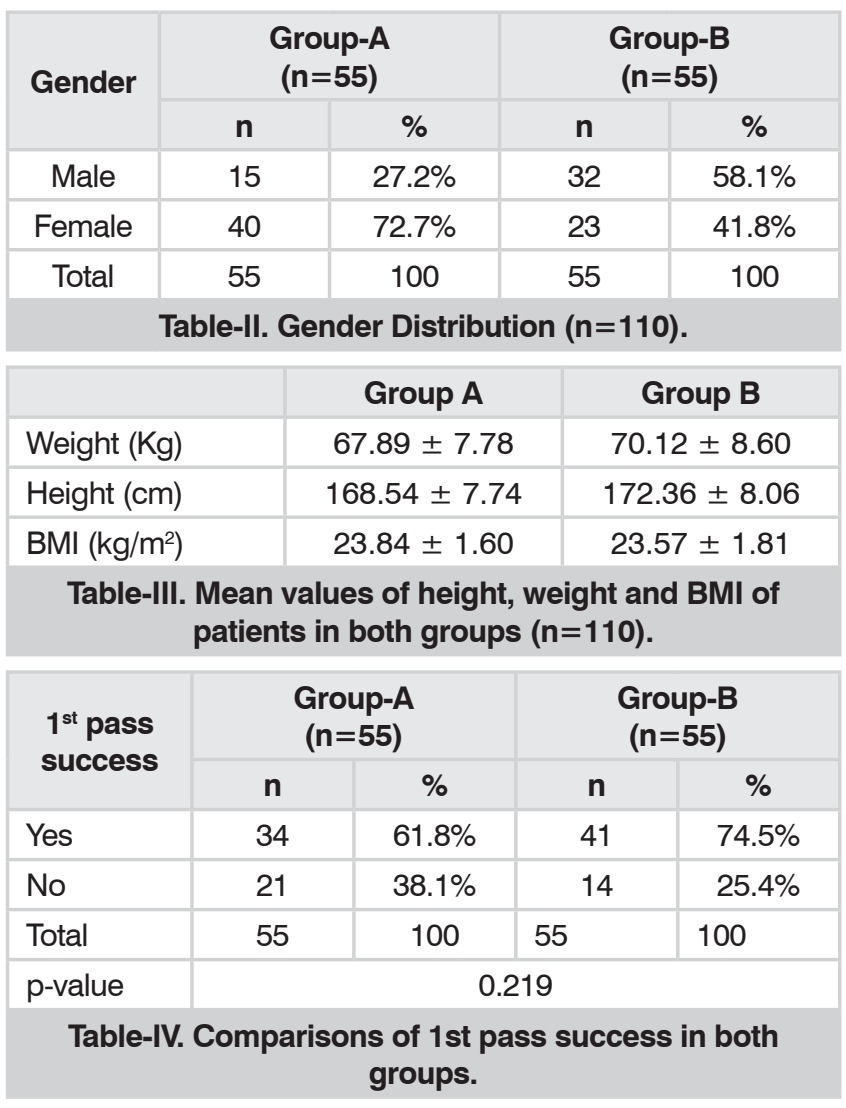

\section{Successful First pass Cannulation - SAX \& LAX}

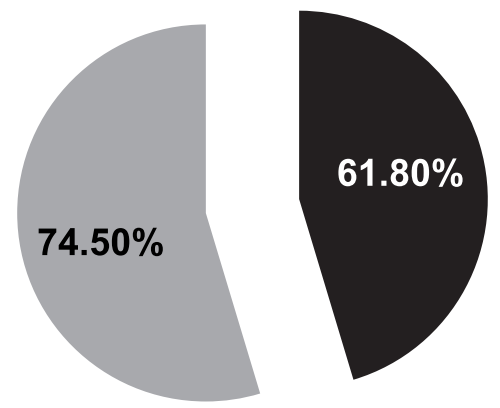

Short-axis view (SAX) Long-axis view (LAX)

Figure-1. Successful first pass cannulation.

\section{DISCUSSION}

Central venous access is an integral part of patient management in critical care. In patients with hemodynamic instabilities ultrasound imaging facilitates IJV cannulation. There are 3 approaches when it comes to US-guided IJV cannulation; short-axis (SAX), long-axis (LAX) and oblique-axis (OAX). SAX has traditionally been the approach preferred by clinicians, however LAX is currently considered to be the recommended approach when performing US-guided vascular access procedures as an in-plane needle insertion can lead to more precise needle tip control. The main objective of the present study was to evaluate the clinical performance of SAX approach vs. LAX approach to ultrasound-guided IJVC. We found that there was no superiority between the SAX and LAX approaches for passing ultrasound guided central line cannulation in IJV. ${ }^{5}$

Numerous studies have compared the SAX and LAX approach to IJ central venous catheterization. Chitoodan et al compared outcomes of the SAX and LAX approach to ultrasound-guided placement of right IJV catheters in patients undergoing cardiac surgery. Patients were randomized to LAX or SAX approach, and each cannulation was performed by anesthetists with experience with $>50$ ultrasound-guided IJV cannulations. The investigators found that the first pass success rate was higher $(p<0.006)$ and fewer needle passes $(p<0.004)$ were necessary in the SAX group as compared to the LAX group. The authors acknowledged that the anesthetists who participated in the study had less experience with the LAX approach to cannulation as compared to the SAX approach. ${ }^{3}$

Several investigators have advocated for the LAX approach to cannulation of the basilic, cephalic, and axillary veins. The authors of these investigations indicate that the LAXapproach offers the unique advantage of continuous visualization of the needle and improved visualization of deeper vessels. Stone et al found that the LAX approach to peripheral venous access afforded increased visibility of the needle tip at the time of vessel puncture as compared to the SAX approach. The LAX view offers the advantage of real-time visualization of the tip of the needle and visualization of the anatomic structure of the target vessel which can be particularly beneficial in cases of anomalous anatomy. ${ }^{6}$ 
Although the SAX approach to IJV catheterization allows visualization of the IJV and its relationship with the carotid, unless the ultrasound operator uses a proper triangulation technique in the SAX to visualize the needle, the needle shaft can be mistaken for the needle tip. In these circumstances, with the needle tip outside the view, the operator may inadvertently enter the carotid or structures posterior to the target vessel such as the lung. Due to the difficulties associated with continuous visualization of the needle tip in the SAX, the ultrasound operator may not realize he or she has punctured the posterior wall of the target vessel. ${ }^{7}$

Blaivas et al conducted a prospective randomized blinded study of resident physicians, and found that in the SAX approach to the IJV, inadvertent PWP occurred in the majority of catheterization attempts and the ultrasound operator was unaware of this outcome. The authors of the study suggested that ultrasound operators be particularly cautious about the location of the needle tip when visualizing the vessel in the SAX or cross-sectional approach. Blaivas and his colleagues outlined several advantages of the LAX approach to vessel cannulation, including continuous visualization of the needle and tip along with the theoretical advantage of not having inadvertent PWP and resultant damage to the structures posterior to the target vessel. ${ }^{8}$ This is especially important since the carotid may lie immediately posterior to the IJV and the lung parenchyma is posterior to the SC access site. Resnick et al also recommend using the LAX to confirm both entry of the needle and angiocatheter into the vessel as well as to assess the length of the catheter within the vessel to ensure appropriate placement and reduce the potential risk of dislodgement. ${ }^{9}$

The LAX approach to vessel cannulation affords unique advantages, but maintaining the needle in the plane of the ultrasound beam may be challenging, especially for novice ultrasound operators. Shofer and his colleagues propose a step-wise approach to maintaining needle visualization in the plane of the ultrasound beam. The steps include obtaining the LAX view, stabilizing the transducer with the non-dominant hand, placing the tip of the needle in the middle of the transducer footprint, inserting the needle, applying the transducer to visualize the needle tip, and advancing the needle to successful cannulation. Shofer and his colleagues indicate that using this approach, the LAX approach has become the preferred method of visualization of vessels for central venous catheterization at their institution. ${ }^{10}$

There are some limitations with the LAX view. The LAX approach may not be feasible in certain anatomic types, such as short neck, which does not allow use of the probe in the vertical orientation necessary for the LAX view. At the IJV site, the LAX view does not afford continuous visualization of the anatomic relationship between the carotid and the IJV. Given this, for the IJV catheterization the ultrasound operator could begin with the SAX approach to correctly identify the vessel which is the IJV. The ultrasound operator can then rotate the probe to the LAX view to facilitate cannulation of the IJV. It may be more difficult for a novice ultrasound user to maintain the probe in the appropriate position to maintain the needle in plane for the LAX visualization. However, if ultrasound education during residency training and at conferences included instruction in the advantages and use of LAX for central venous catheterization, users may become more facile with use of the LAX view. ${ }^{11}$

In spite of our limitations, Gentle Sunder Shrestha, Arjun Gurung, Sabin Koirala also second our finding by stating that both techniques have similar outcomes when used for IJV vein cannulation. The ultrasound probe used in their study was a 6-10 L38 MHz linear transducer SonoSite turbo unit (SonoSite ${ }^{\circledR}$, Micromaxx, Bothwell, WA, USA). Their sample size was 82 with first pass success in short axis was $67.9 \%$ while in long axis its $51.2 \%$ which is comparable with our results. ${ }^{12}$

M. Batllori et al conducted an RCT where 220 patients were randomized and univariate analysis demonstrated an overall cannulation success rate in designated approaches was $96.4 \%$, with no differences between groups. However, first needle 
pass cannulations differed between groups in a univariate comparison, and a multivariate analysis confirmed that the success rate of first needle passes was lower in LAX than in both OAX (OR 3.70; 95\% Cl 1.71, 8.0) and SAX (OR 2.37; $95 \% \mathrm{Cl} 1.16,4.86)$. No significant differences were observed between SAX and OAX. Patient characteristics and baseline characteristics did not differ significantly between study groups. ${ }^{5}$

Similarly six randomized controlled trials with 621 patients showed no significant differences in total success rate and first-pass success rate, as well as in the arterial puncture, hematoma, or catheterrelated bloodstream infection complications between SAX and LAX approaches. Moreover, no significant difference was found between SAX and OAX approaches in terms of total success rate, first-pass success rate, number of needle passes, and complications of arterial puncture and hematoma. However, the number of needle passes was significantly fewer in SAX approach than in LAX approach (weighted mean difference,-0.18; 95\% confidence interval,-0.35 to0.01). Since none of the scanning axes exhibited unique features that could enhance their suitability for application. Hence, scanning axes should be selected by considering various factors that include patient characteristics, clinician expertise, and ease of procedures. ${ }^{13}$

\section{CONCLUSION}

There is no significant difference in first pass success rate between long (LAX) and short axis (SAX) approaches in US-guided IJV cannulation. Thus, this study concluded that both approaches have equal first pass success rate.

\section{Copyright $\subset$}

\section{REFERENCES}

1. Yang EJ, Ha HS, Kong YH, Kim SJ. Ultrasound-guided internal jugular vein catheterization in critically ill pediatric patients. Korean journal of pediatrics. 2015; 58(4):136-41.
2. Troianos CA, Hartman GS, Glas KE, Skubas NJ, Eberhardt RT, Walker JD, Reeves ST. Guidelines for performing ultrasound guided vascular cannulation: recommendations of the American Society of Echocardiography and the Society of Cardiovascular Anesthesiologists. Journal of the American Society of Echocardiography. 2011 Dec 1;24(12):1291-318. doi: 10.1016/j.echo.2011.09.021

3. Chittoodan S, Breen D, D O'Donnell B, lohom G. Long versus short axis ultrasound guided approach for internal jugular vein cannulation: a prospective randomised controlled trial. Medical ultrasonography. $2011 ; 13(1): 21$.

4. Vogel JA, Haukoos JS, Erickson CL, Liao MM, Theoret J, Sanz GE, et al. Is long-axis view superior to shortaxis view in ultrasound-guided central venous catheterization? Critical care medicine. 2015;43(4):8329.

5. Batllori M, Urra M, Uriarte E, Romero C, Pueyo J, López-Olaondo L, Cambra K, Ibáñez B. Randomized comparison of three transducer orientation approaches for ultrasound guided internal jugular venous cannulation. BJA: British Journal of Anaesthesia. 2016 Mar 1;116(3):370-6.

6. Randolph AG, Cook DJ, Gonzales CA, Pribble CG. Ultrasound guidance for placement of central venous catheters: a meta-analysis of the literature. Critical care medicine. 1996; 24(12):2053-8.

7. DiLisio R, Mittnacht AJ. The "medial-oblique" approach to ultrasound-guided central venous cannulation-M aximize the view, minimize the risk. Journal of cardiothoracic and vascular anesthesia. 2012; 26(6):982-4.

8. Blaivas M, Adhikari S. An unseen danger: frequency of posterior vessel wall penetration by needles during attempts to place IJV central catheters using ultrasound guidance. Critical care medicine. 2009; $37(8): 2345-9$.

9. Resnick JR, Cydulka R, Jones R. Comparison of two transducers for ultrasound-guided vascular access in long axis. The Journal of emergency medicine. 2007; 33(3):273-6.

10. Davis PJ, Cladis FP, Motoyama EK. Smith's Anesthesia for Infants and Children E-Book: Expert Consult Premium: Elsevier Health Sciences; 2010. 
11. Airapetian N, Maizel J, Langelle F, Modeliar SS, Karakitsos D, Dupont $\mathrm{H}$, et al. Ultrasound-guided central venous cannulation is superior to quicklook ultrasound and landmark methods among inexperienced operators: a prospective randomized study. Intensive care medicine. 2013; 39 (11):1938-44.

12. Shrestha GS, Gurung A, Koirala S. Comparison between long-and short-axis techniques for ultrasound-guided cannulation of internal jugular vein. Annals of cardiac anaesthesia. 2016; 19 (2):288.
13. Chen, J. Y., Wang, L. K., Lin, Y. T., Lan, K. M., Loh, E. W., Chen, C. H., \& Tam, K. W. (2019). Comparing short-, long-, and oblique-axis approaches to ultrasoundguided internal jugular venous catheterization: $A$ meta-analysis of randomized controlled trials. Journal of Trauma and Acute Care Surgery, 86(3), 516-523. https:// doi.org/10.1097/TA.0000000000002158.

\begin{tabular}{|c|c|c|c|}
\hline \multicolumn{4}{|c|}{ AUTHORSHIP AND CONTRIBUTION DECLARATION } \\
\hline Sr. \# & Author(s) Full Name & Contribution to the paper & Author(s) Signature \\
\hline 1 & Shaheer Nayyar & $\begin{array}{l}\text { Principal author, Study design, } \\
\text { Study conduct, data analysis, } \\
\text { Paper writing. } \\
\text { Study conduct \& data collection. }\end{array}$ & \\
\hline 3 & Rizwan Ahmad Khan & Study conduct. & \\
\hline 4 & Hajra Shuja & Data analysis. & \\
\hline 5 & Irfan Liaqat Ali & Data analysis \& paper writing. & \\
\hline 6 & Muhammad Ashraf Zia & Review of study. & \\
\hline
\end{tabular}

